\title{
Jugular Bulb Diverticulum: A Rare Cause of Pulsatile Tinnitus and Literature Review
}

\author{
Tzu-I Wu1 ${ }^{1}$ Hsing-Mei Wu $\mathbf{u}^{1,2 *}$ \\ ${ }^{1}$ Department of Otolaryngology Head and Neck Surgery, Shin Kong Wu-Ho-Su Memorial Hospital, Taiwan \\ ${ }^{2}$ School of Medicine, Fu-Jen Catholic University, Taiwan \\ Email: ^hsingmeiwu@yahoo.com
}

How to cite this paper: $\mathrm{Wu}, \mathrm{T}$.- $\mathrm{I}$ and $\mathrm{Wu}$, H.-M. (2021) Jugular Bulb Diverticulum: A Rare Cause of Pulsatile Tinnitus and Literature Review. International Journal of Otolaryngology and Head \& Neck Surgery, 10, 359-364.

https://doi.org/10.4236/ijohns.2021.105032

Received: July 2, 2021

Accepted: August 22, 2021

Published: August 25, 2021

Copyright $\odot 2021$ by author(s) and Scientific Research Publishing Inc. This work is licensed under the Creative Commons Attribution International License (CC BY 4.0).

http://creativecommons.org/licenses/by/4.0/

\section{Open Access}

\begin{abstract}
Objectives: Pulsatile tinnitus involves a wide spectrum of etiologies. The etiologies include normal vascular variants, temporal bone tumor, acquired vascular lesions and chronic middle ear inflammatory diseases. Jugular bulb diverticulum is a rare cause of pulsatile tinnitus. We report one case of jugular diverticulum presenting with pulsatile tinnitus and its surgical management and outcome. Case Report: A 36-year-old woman with a history of uterine myoma and chronic anemia presented with right pulsatile tinnitus that was worsening in recent one month. Neurological exam was normal. ENT evaluation revealed no abnormalities via otoscopy and physical examination. The CT scan revealed right dominant jugular bulb with diverticulum projecting to posterior ear canal wall. We performed jugular bulb diverticulum resurfacing with temporalis fascia, Surgicel ${ }^{\circledR}$ and Gelfoam ${ }^{\circledR}$, and bone wax via transmastoid approach. The symptom improved postoperative immediately. No major complications were noted during outpatient clinic follow-up. Conclusion: Transmastoid resurfacing of jugular bulb diverticulum is an effective management of pulsatile tinnitus from this kind vascular anomaly.
\end{abstract}

\section{Keywords}

Pulsatile Tinnitus, Jugular Bulb Anomaly, Jugular Bulb Diverticulum

\section{Introduction}

Tinnitus is the perception of hearing a sound when no external sound is present. It may be classified as subjective versus objective, as well as pulsatile versus continuous. Pulsatile tinnitus is a rhythmic sound which is cardiac synchronous and most cases are vascular origin. Patients present with pulsatile tinnitus must be carefully evaluated as some may be diagnosed with a treatable lesion. A retros- 
pective study reviewing 84 patients with pulsatile tinnitus seen in a neurology department, 42 percent were found to have a significant vascular disorder [1]. Jugular bulb abnormality such as jugular bulb diverticulum, a protrusion of the jugular bulb with waist-like margin, can result in vascular tinnitus [2] [3]. In this report, we share our experience in surgically treating a case of jugular bulb diverticulum with a transmastoid approach.

\section{Case Report}

36-year-old woman with a history of uterine myoma and chronic anemia presented with a month history of right pulsatile tinnitus that was worsening. The patient denied other associated symptoms such as dizziness, vertigo, headache, aural fullness, or apparent hearing impairment. Neurological exam was normal. ENT evaluation revealed no abnormalities via otoscopy and physical examination, but pure-tone audiometry revealed a right low-tone sensorineural hearing loss.

A temporal bone computed tomography (CT) scan showed an enlarged right jugular foramen with upward osteolytic lesion projecting just posterior to the IAC (Figure 1). With possible differential diagnoses of large jugular bulb with diverticulum or tumor lesion in mind, magnetic resonance angiography was done and showed no definite glomus tumor while confirming an engorged and high right jugular bulb with diverticulum (Figure 2).

Under the diagnosis of right large jugular bulb with diverticulum, the patient was treated surgically. Cortical mastoidectomy was performed to expose the jugular diverticulum with assistance of the StealthStation ${ }^{\mathrm{TM}}$ S8 surgical navigation system. The facial nerve was not exposed but located with nerve monitor and avoided. The diverticulum was exposed after drilling out of the retrofacial aircells (Figure 3). After careful removal of the thin bony cortex covering the diverticulum, the bony defect was packed with temporalis fascia, Surgicel ${ }^{\circledR}$, Gelfoam ${ }^{\circledR}$, bone wax, and fibrin glue (Tisseel ${ }^{\circledR}$ ) to create a smooth resurfacing of the bony wall (Figure 4). Compressive mastoid dressing was applied after the wound was closed in two layers, and patient put on bedrest for 24 hours postoperatively.

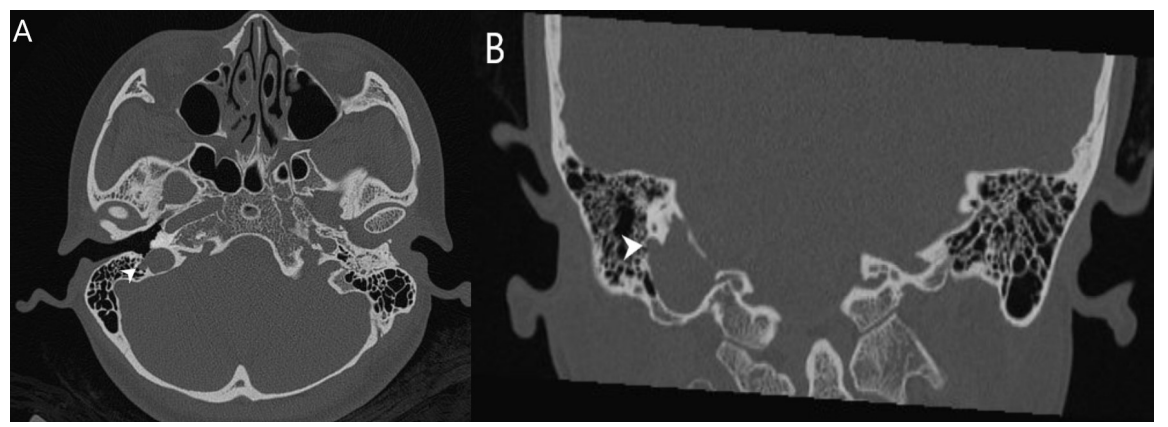

Figure 1. High resolution computed tomography of temporal bone revealing enlarged right jugular foramen with upward osteolytic projecting (white arrow; (A) Sagittal, (B) Coronal). 


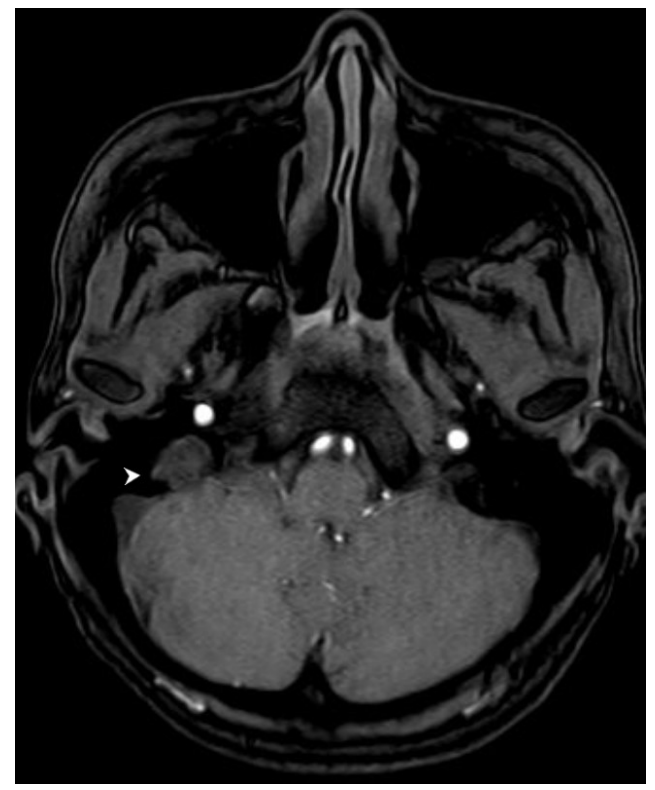

Figure 2. Magnetic resonance imaging of gadolinium enhanced T1 weighted series with fat suppression showing engorged and high right jugular bulb without evidence of tumor lesion (White arrow).

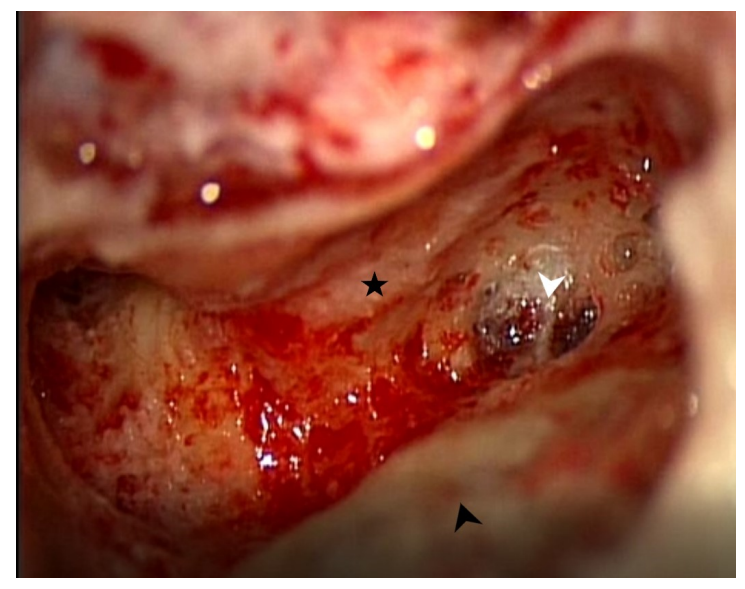

Figure 3. The diverticulum (white arrow) was exposed after drilling out of the retrofacial aircells (Black arrow: sigmoid sinus, black star: location of facial nerve).

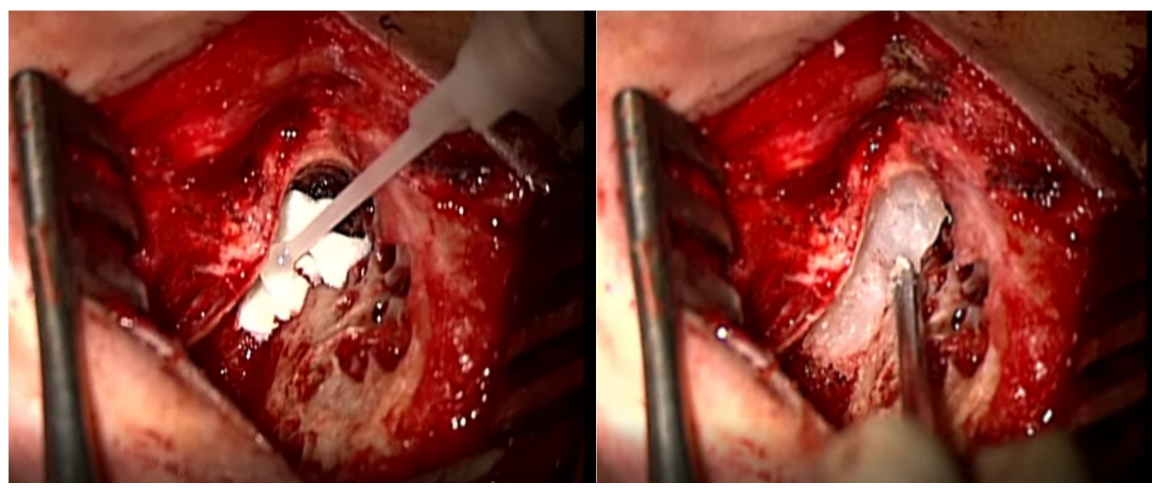

Figure 4. The diverticulum was packed with temporalis fascia, Surgicel ${ }^{\circledR}$, Gelfoam ${ }^{\circledR}$, bone wax, Tisseel ${ }^{\circledR}$, and bone wax to provide a smooth resurfacing of the bony wall. 
The postoperative course was uneventful, and the patient experienced complete regression of the pulsatile tinnitus postoperative immediately. Pure tone audiometry tested three weeks postoperatively showed no hearing impairment. Subjective improvement of pulsatile tinnitus was confirmed at subsequent follow-up visits at the outpatient clinic and no relapse noted as far as the last follow-up in June of 2020, three months after surgical treatment.

\section{Discussion}

Vascular tinnitus is the one of the most common form of pulsatile tinnitus, which is defined as patient complaining hearing a repetitive sound that is synchronous with the patient's pulse [4]. It is frequently associated to a turbulent blood flow transmitted at the middle or inner ear, secondary to the presence of vascular lesions [5] [6]. Jugular bulb diverticulum is a clinical entity that may be the cause of vascular tinnitus due to results of turbulent blood flows caused by venous sinus stenosis near the middle ear [1] [2] [3] [4] [5]. This is supported by data showing the lesion is located in the dominant sinus in $84.6 \%$ of patients [7].

The diagnosis of vascular tinnitus is challenging due to its rarity and lack standardized diagnostic protocol [6]. However, imaging studies are crucial to the diagnosis of jugular bulb abnormalities. High-resolution CT of the temporal bone is widely accepted to be the initial imaging study for pulsatile tinnitus, as it is capable of reliably identifying the detailed anatomy and pathology of the temporal bone, and is considered best imaging modality for evaluation of high-riding jugular bulb. MRA is particularly useful in detecting arterial origins of vascular tinnitus, but veins and sinuses are better represented by CT angiography [5] [6].

Both surgery and endovascular procedures have been reported as treatment of jugular bulb diverticulum. Literatures of successful treatment by endovascular approaches are limited to case reports or series. Stenting and coiling are utilized in order to occlude the diverticulum while preserving the venous drainage pattern [7] [8] [9]. Associated risks of endovascular procedure include stent or vein thrombosis or coil migration, which may result in intracranial hypertension, cerebral venous ischemia, superior vena cava syndrome and even pulmonary embolism secondary to dislodgment of clot. Sanchez et al. reported a small area of cerebellar ischemia after embolization of a sigmoid sinus diverticulum [10]. However, the advantages of endovascular treatment are its minimally invasive nature, which allows for convenient repeated treatment should the need arises, and also allowing the procedure to be done in an awake fashion making immediate confirmation of treatment efficacy possible as described by Park et al. [11].

There are few literatures on the surgical management of jugular divertivulum. A retrospective study of 13 cases by V. Couloigner published in 1998 reported surgically lowering of the jugular bulb with surgical wax surrounded by Surgicel helped three (23\%) patient to experience total disappearance of tinnitus, and reduced number patients with disabling tinnitus from nine (69\%) to four (31\%) [12]. Otto KJ et al. first described a series of three cases that successfully under- 
went transmastoid reconstruction of the sigmoid sinus diverticulum [13]. The surgery was designed to reduce the diverticulum and reconstruct the wall of the sigmoid sinus, in order to provide a smooth internal lumen. He also utilized bone wax along with temporalis fascia/muscle as the material of choice [13]. Recently Yeo et al. reported two cases of jugular bulb diverticulum successfully treated the same transmastoid surgical approach, and resurfaced the bony defect with temporalis muscle fascia and concha cartilage [14]. In our case, we opted to use temporalis fascia, Surgicel ${ }^{\circledR}$, Gelfoam $^{\circledR}$, bone wax, and fibrin glue (Tisseel ${ }^{\circledR}$ ) for reconstructing the bony defect. Bone wax is easy to manipulate into exact shapes and sizes, while highly accessible.

Evidences of successful endovascular and surgical management of pulsatile tinnitus caused by jugular abnormalities are limited by short follow up times, with most reporting data with follow-up periods of less than one year. There is also a lack of randomized control data support. However, based on our experience and literature review, surgical management can be a promising option for patients with pulsatile tinnitus caused by jugular bulb diverticulum.

\section{Conclusion}

We report a case of pulsatile tinnitus caused by jugular bulb diverticulum treated by surgical management. By reconstructing and resurfacing the bony defect surrounding the diverticulum, surgery is able to provide an effective outcome. No major or long term complications were encountered in our case.

\section{Financial Support}

This research received no specific grant from any funding agency, commercial or not-for-profit sectors.

\section{Ethical Standards}

The authors assert that all procedures contributing to this work comply with the ethical standards of the Shin-Kong Wu-Ho-Su Memorial Hospital guidelines on human experimentation and with the Helsinki Declaration of 1975, as revised in 2008.

\section{Conflicts of Interest}

The authors declare no conflicts of interest regarding the publication of this paper.

\section{References}

[1] Waldvogel, D., Mattle, H.P., Sturzenegger, M. and Schroth, G. (1998) Pulsatile Tinnitus-A Review of 84 Patients. Journal of Neurology, 245, 137-142. https://doi.org/10.1007/s004150050193

[2] El-Kashlan, H.K., Arts, H.A. and Gebarski, S. (2000) Jugular Diverticulum: Clinical Significance. Otolaryngology_Head and Neck Surgery, 122, 575-576.

[3] Grewal, A.K., Kim, H.Y., Comstock, R.H., et al. (2014) Clinical Presentation and 
Imaging Findings in Patients with Pulsatile Tinnitus and Sigmoid Sinus Diverticulum/Dehiscence. Otology \& Neurotology, 35, 16-21. https://doi.org/10.1097/MAO.0b013e31829ab6d7

[4] Stouffer, J.L. and Tyler, R.S. (1990) Characterization of Tinnitus by Tinnitus Patients. The Journal of Speech and Hearing Disorders, 55, 439-453.

[5] Hofmann, E., Behr, R., Neumann-Haefelin, T., et al. (2013) Pulsatile Tinnitus: Imaging and Differential Diagnosis. Deutsches Ärzteblatt International, 110, 451-458.

[6] Bae, S.C., Kim, D.K., Yeo, S.W., Park, S.Y. and Park, S.N. (2015) Single-Center 10-Year Experience in Treating Patients with Vascular Tinnitus: Diagnostic Approaches and Treatment Outcomes. Clinical and Experimental Otorhinolaryngolo$g y$, 8, 7-12. https://doi.org/10.3342/ceo.2015.8.1.7

[7] Trivelato, F.P., Araújo, J.F., Dos Santos Silva, R., Rezende, M.T., Ulhôa, A.C. and Castro, G.D. (2015) Endovascular Treatment of Pulsatile Tinnitus Associated with Transverse Sigmoid Sinus Aneurysms and Jugular Bulb Anomalies. Interventional Neuroradiology, 21, 548-551. https://doi.org/10.1177/1591019915590367

[8] Mehanna, R., Shaltoni, H., Morsi, H. and Mawad, M. (2010) Endovascular Treatment of Sigmoid Sinus Aneurysm Presenting as Devastating Pulsatile Tinnitus. A Case Report and Review of Literature. Interventional Neuroradiology, 16, 451-454. https://doi.org/10.1177/159101991001600413

[9] Hui, F.K., Abruzzo, T. and Ansari, S.A. (2016) Endovascular Interventions for Idiopathic Intracranial Hypertension and Venous Tinnitus: New Horizons. Neuroimaging Clinics of North America, 26, 289-299. https://doi.org/10.1016/j.nic.2015.12.007

[10] Sanchez, T.G., Murao, M., de Medeiros, I.R., et al. (2002) A New Therapeutic Procedure for Treatment of Objective Venous Pulsatile Tinnitus. The International Tinnitus Journal, 8, 54-57.

[11] Park, Y.H. and Kwon, H.J. (2011) Awake Embolization of Sigmoid Sinus Diverticulum Causing Pulsatile Tinnitus: Simultaneous Confirmative Diagnosis and Treatment. Interventional Neuroradiology, 17, 376-379. https://doi.org/10.1177/159101991101700316

[12] Couloigner, V., Grayeli, A.B., Bouccara, D., Julien, N. and Sterkers, O. (1999) Surgical Treatment of the High Jugular Bulb in Patients with Ménière's Disease and Pulsatile Tinnitus. European Archives of Oto-Rhino-Laryngology, 256, 224-229. https://doi.org/10.1007/s004050050146

[13] Otto, K.J., Hudgins, P.A., Abdelkafy, W., et al. (2007) Sigmoid Sinus Diverticulum: A New Surgical Approach to the Correction of Pulsatile Tinnitus. Otology \& Neurotology, 28, 48-53. https://doi.org/10.1097/01.mao.0000247814.85829.f6

[14] Yeo, W.X., Xu, S.H., Tan, T.Y., Low, Y.M. and Yuen, H.W. (2018) Surgical Management of Pulsatile Tinnitus Secondary to Jugular Bulb or Sigmoid Sinus Diverticulum with Review of Literature. American Journal of Otolaryngology, 39, 247-252. https://doi.org/10.1016/j.amjoto.2017.12.019 